\title{
ANTI-SLUG CONTROL OF GAS-LIFT WELLS - EXPERIMENTAL RESULTS ${ }^{1}$
}

\author{
Gisle Otto Eikrem*,2 Ole Morten Aamo* \\ Hardy Siahaan* Bjarne Foss* \\ * Department of Engineering Cybernetics \\ Norwegian University of Science and Technology \\ 7491 Trondheim, Norway
}

\begin{abstract}
Unstable flow regimes occurring for multiphase flow in gas-lift wells have successfully been stabilized using conventional linear control techniques. However, the control systems rely on downhole measurements which are at best unreliable, if at all available. In this paper, we employ a nonlinear observer for the states of the multiphase flow in the gas-lift well. The observer relies on topside measurements only, and downhole pressure for feedback control is estimated. Combining the nonlinear observer with conventional PI control of the downhole pressure, we demonstrate in laboratory experiments the potential for increasing production from gas-lift oil wells by stabilizing the multiphase flow.
\end{abstract}

Keywords: Multiphase, Observer, Experiments, Gas-Lift

\section{INTRODUCTION}

This paper investigates the possibility of using a nonlinear observer for estimation of the downhole pressure of a gas-lift well. The estimated downhole pressure will rely only on topside measurements, and will replace the direct measurement of the downhole pressure, as input to the stabilizing controller.

The objective is to increase the production of liquid from the gas-lift well. The method is to reduce friction by increasing the opening of the production choke. The increased production choke opening may cause unstable production, which can be handled by the use of feedback control.

\footnotetext{
1 This work was supported by the Gas Technology Center and Petronics at NTNU, and the Norwegian Research Council.

2 Author to whom correspondence should be addressed. E-mail: Gisle.Otto.Eikrem@itk.ntnu.no
}

\section{GAS-LIFT WELL}

The use of control in multiphase flow systems is an area of increasing interest to the oil and gas industry. Oil wells with highly oscillatory flow are a significant problem in the petroleum industry. Several different instability phenomena related to oil and gas wells exist, and in this study, highly oscillatory flow in gas-lift wells will be the area of investigation. Gas lift is a technology to produce oil and gas from wells with low reservoir pressure by reducing the hydrostatic pressure in the tubing. Gas is injected into the tubing, as deep as possible, and mixes with the fluid from the reservoir, see Figure 1.

The gas reduces the density of the fluid in the tubing, which in turn reduces the downhole pressure (DHP), and thereby increases the production from the reservoir. The lift gas is routed from the surface and into the annulus, which is the volume between the casing and the tubing. The gas enters the tubing through a gas injection valve, which 


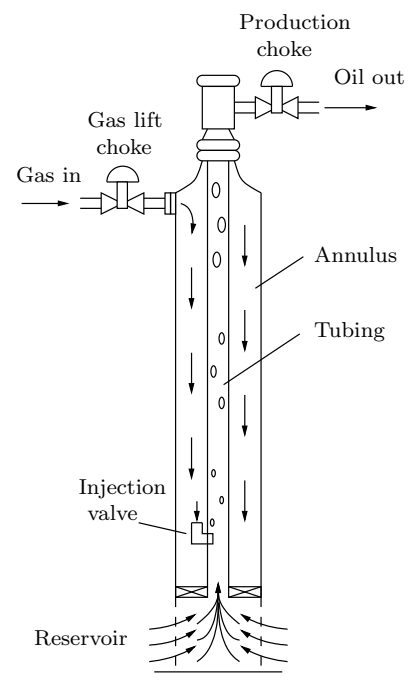

Fig. 1. A gas lifted oil well

does not permit backflow from the tubing and into the annulus. The dynamics of highly oscillatory flow in gas-lift wells can be described as follows:

(1) Gas from the annulus starts to flow into the tubing. As gas enters the tubing the pressure in the tubing falls. This accelerates the inflow of gas.

(2) The gas pushes the major part of the liquid out of the tubing.

(3) Liquid in the tubing generates a blocking constraint downstream the gas injection valve. Hence, the tubing gets filled with liquid and the annulus with gas.

(4) When the pressure upstream of the gas injection valve dominates the pressure on the downstream side, a new cycle starts.

For more information on this type of instability, often termed severe slugging, see $\mathrm{Xu}$ and Golan (1999).

To avoid the oscillatory behaviour of the gaslift oil wells, continuous flow of gas from the annulus to the tubing is required. The purpose of all stabilizing methods is to maintain this continuous gas flow into the tubing. When the pressure drop of the fluid flow in the system is friction dominated, the system is stable. If the pressure drop is gravity dominated the system is unstable, and control is required.

There are two methods to eliminate this highly oscillatory well flow in the gas-lift wells: The first method is to increase the pressure drop caused by friction either by increasing the gas flow rate, reducing the opening of the production choke or reducing the size of the gas injection valve. The second method is the use of feedback control to stabilize the well flow, which is the subject of this study. Figure 2 shows a conceptual gas lift production curve. The produced oil rate is a function of the flow rate of gas injected into the well.
The curve shows under which conditions the well exhibits stable or highly oscillatory flow. It is important to note that the average production rate may be significantly lower with unstable, see the line labeled "open loop production", compared to stable well flow, see the line labeled "theoretical production". In addition to causing lower oil production, the large oscillations in the flow rate from the well causes topside production problems like poor downstream oil/water separation and flaring. The region of optimum lift gas utilization may lie in the unstable region, since the use of lift gas is associated with a cost and since the gas injection rate may be upper bounded.

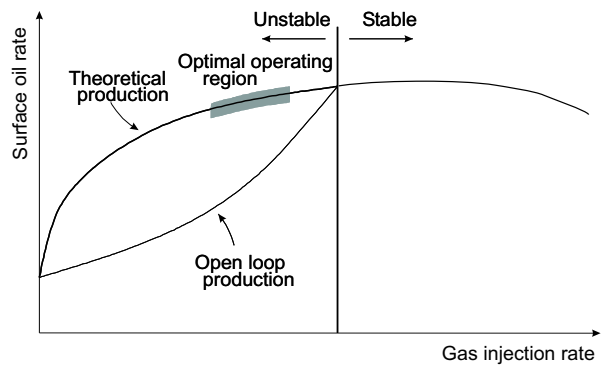

Fig. 2. The gas lift curve with the region of optimum lift gas utilization.

Stabilization of gas-lift wells using conventional control techniques has been studied for single well systems in Dalsmo et al. (2002), Jansen et al. (1999) and Kinderen and Dunham (1998); and for a two-well system in Eikrem et al. (2002). In Imsland (2002), a state feedback control law was designed using Lyapunov theory. And this controller was used in an output feedback setting with an extended Kalman filter in Eikrem et al. (2004). In this paper, we employ a nonlinear observer for the states of the multiphase flow in the gas-lift well, and estimate downhole pressure for feedback control. The observer-controller scheme is tested in laboratory experiments.

\section{MATHEMATICAL MODEL}

The process described in Section 2, and sketched in Figure 1, is modelled by three states: $x_{1}$ is the mass of gas in the annulus, $x_{2}$ is the mass of gas in the tubing and $x_{3}$ is the mass of oil in the tubing. Looking at Figure 1, we have

$$
\begin{aligned}
& \dot{x}_{1}=w_{g c}-w_{i v}, \\
& \dot{x}_{2}=w_{i v}-w_{p g}, \\
& \dot{x}_{3}=w_{r}-w_{p o},
\end{aligned}
$$

where $w_{g c}$ is a constant mass flow rate of lift gas injected into the annulus, $w_{i v}$ is the mass flow rate of lift gas from the annulus and into the tubing, $w_{p g}$ is the mass flow rate of gas through the production choke, $w_{r}$ is the oil mass flow rate from the reservoir into the tubing, and $w_{p o}$ is 
the mass flow rate of produced oil through the production choke. The flows are modelled by:

$$
\begin{aligned}
w_{g c} & =\text { constant flow rate of lift gas, } \\
w_{i v} & =C_{i v} \sqrt{\rho_{a, i} \max \left\{0, p_{a, i}-p_{t, i}\right\}}, \\
w_{p c} & =C_{p c} \sqrt{\rho_{m} \max \left\{0, p_{t}-p_{s}\right\}} u, \\
w_{p g} & =\frac{x_{2}}{x_{2}+x_{3}} w_{p c}, \\
w_{p o} & =\frac{x_{3}}{x_{2}+x_{3}} w_{p c}, \\
w_{r} & =C_{r}\left(p_{r}-p_{t, b}\right) .
\end{aligned}
$$

$C_{i v}, C_{p c}$, and $C_{r}$ are constants, $u$ is the production choke opening $(u(t) \in[0,1]), \rho_{a, i}$ is the density of gas in the annulus at the injection point, $\rho_{m}$ is the density of the oil/gas mixture at the top of the tubing, $p_{a, i}$ is the pressure in the annulus at the injection point, $p_{t, i}$ is the pressure in the tubing at the gas injection point, $p_{t}$ is the pressure at the top of the tubing, $p_{s}$ is the pressure in the separator, $p_{r}$ is the pressure in the reservoir, and $p_{t, b}$ is the pressure at the bottom of the tubing. The separator pressure, $p_{s}$, is assumed to be held constant by a control system, and the reservoir pressure, $p_{r}$, is assumed to be slowly varying and therefore treated as constant. Note that flow rates through the valves are restricted to be positive. The densities are modelled as follows

$$
\begin{aligned}
\rho_{a, i} & =\frac{M}{R T_{a}} p_{a, i}, \\
\rho_{m} & =\frac{x_{2}+x_{3}-\rho_{o} L_{r} A_{r}}{L_{t} A_{t}},
\end{aligned}
$$

and the pressures as follows

$$
\begin{aligned}
p_{a, i} & =\left(\frac{R T_{a}}{V_{a} M}+\frac{g L_{a}}{V_{a}}\right) x_{1}, \\
p_{t} & =\frac{R T_{t}}{M} \frac{x_{2}}{L_{t} A_{t}+L_{r} A_{r}-\nu_{o} x_{3}}, \\
p_{t, i} & =p_{t}+\frac{g}{A_{t}}\left(x_{2}+x_{3}-\rho_{o} L_{r} A_{r}\right), \\
p_{t, b} & =p_{t, i}+\rho_{o} g L_{r} .
\end{aligned}
$$

$M$ is the molar weight of the gas, $R$ is the gas constant, $T_{a}$ is the temperature in the annulus, $T_{t}$ is the temperature in the tubing, $V_{a}$ is the volume of the annulus, $L_{a}$ is the length of the annulus, $L_{t}$ is the length of the tubing, $A_{t}$ is the cross sectional area of the tubing above the injection point, $L_{r}$ is the length from the bottom of the well to the gas injection point, $A_{r}$ is the cross sectional area of the tubing below the injection point, $g$ is the gravity constant, $\rho_{o}$ is the density of the oil, and $\nu_{o}$ is the specific volume of the oil. The oil is considered incompressible, so $\rho_{o}$ is constant. The temperatures, $T_{a}$ and $T_{t}$ are slowly varying and therefore treated as constants.

\section{STATE ESTIMATION}

The downhole pressure is often a measurement which is not available. This measurement is suit- able for stabilization of an unstable multiphase flow system like the gas-lift well. The objective of the observer is to calculate an estimate of the downhole pressure, to be used as controller input.

We will in this work assume that we have measurements at the top of the annulus and tubing, only. The main challenge is how to deal with the multiphase flow in the tubing, whereas the single phase flow in the annulus can accurately be estimated based on one pressure measurement and one temperature measurement. Thus, we will assume that $x_{1}$ is measured. For estimation of the two remaining states, we measure the pressure and the density at the top of the tubing. Our measurements are therefore

$$
\begin{gathered}
y_{1}(t)=x_{1}(t), y_{2}(t)=p_{t}(t), \\
y_{3}(t)=\rho_{m}(t) .
\end{gathered}
$$

Since the mass of gas in the annulus can be considered a measurement, a reduced order observer is used for estimation of the remaining two states. Before giving the observer equations, we state key assumptions under which nominal performance of the observer is guaranteed, see also Aamo et al. (2004).

Assumption 1. The production choke is not allowed to close completely. That is,

$$
u \geq \delta_{u}>0, \forall t \geq 0
$$

Assumption 2. The states are bounded away from zero, and the part of the tubing below the gas injection point is filled with oil. More precisely,

$$
\begin{aligned}
& x_{1} \geq \delta_{1}>0, \\
& x_{2} \geq \delta_{2}>0, \\
& x_{3} \geq \rho_{o} L_{r} A_{r}+\delta_{3}>\rho_{o} L_{r} A_{r}, \forall t \geq t_{0} .
\end{aligned}
$$

Assumption 3. The gas in the tubing has lower density than the oil. More precisely,

$$
L_{t} A_{t}+L_{r} A_{r}-\nu_{o}\left(x_{3}+x_{2}\right) \geq \delta_{g}>0, \forall t \geq 0 .
$$

The observer equations are the following (Aamo et al., 2004)

$$
\begin{aligned}
\dot{\hat{z}}_{1} & =w_{g c}-\frac{\hat{z}_{1}-y_{1}}{\hat{z}_{2}-y_{1}} v+k_{1}\left(\hat{z}_{1}, \hat{z}_{2}, y_{1}, y_{2}\right), \\
\dot{\hat{z}}_{2} & =w_{g c}+C_{r}\left(p_{r}-\rho_{o} g L_{r}+\frac{A_{r}}{A_{t}} \rho_{o} g L_{r}\right. \\
& \left.+\frac{g}{A_{t}} y_{1}-y_{2}-\frac{g}{A_{t}} \hat{z}_{2}\right)-v \\
& +k_{2}\left(\hat{z}_{2}, u, v, y_{1}, y_{2}\right) \\
\hat{z}_{1} & \geq \delta_{2}+y_{1}, \text { and } \hat{z}_{2} \geq \rho_{o} L_{r} A_{r}+\delta_{3}+\hat{z}_{1}, \\
\hat{x}_{2} & =\hat{z}_{1}-y_{1}, \\
\hat{x}_{3} & =\hat{z}_{2}-\hat{z}_{1},
\end{aligned}
$$


where $z_{1}$ is the total mass of gas and $z_{2}$ is the total mass of gas and liquid, and

$$
v=C_{p c} \sqrt{y_{3} \max \left\{0, y_{2}-p s\right\}} u .
$$

The output injections, $k_{1}$ and $k_{2}$, are given by

$$
\begin{array}{r}
k_{1}\left(\hat{z}_{1}, \hat{z}_{2}, y_{1}, y_{2}\right)=c_{1}\left(\frac { M } { R T _ { t } } \left(L_{t} A_{t}+L_{r} A_{r}\right.\right. \\
\left.\left.-\nu_{o}\left(\hat{z}_{2}-\hat{z}_{1}\right)\right) y_{2}-\left(\hat{z}_{1}-y_{1}\right)\right), \\
k_{2}\left(\hat{z}_{2}, y_{1}, y_{3}\right)=c_{2}\left(y_{3}-\frac{\hat{z}_{2}-y_{1}-\rho_{o} L_{r} A_{r}}{L_{t} A_{t}}\right) .
\end{array}
$$

where $c_{1}$ and $c_{2}$ are arbitrary positive constants. The convergence rate of the observer increases with increasing $c_{1}$ and $c_{2}$.

\section{ANTI-SLUG CONTROL BY FEEDBACK}

It has been shown in Eikrem et al. (2002) that severe slugging can be eliminated by stabilizing the downhole pressure using a control law of the form

$$
v=v^{*}+K\left(p_{t, b}^{*}-p_{t, b}\right),
$$

where $v^{*}$ and $p_{t, b}^{*}$ are some appropriate constants.

We will apply the observer described in Section 4 to obtain an estimate for the downhole pressure of the gas-lift well. This observer is applied in the control structure given in Figure 3, and will generate an estimate of $p_{t, b}$, see (15).

For linear systems, stability of the closed loop system using the certainty equivalence controller is guaranteed by the separation principle of linear systems. For general nonlinear systems, however, not even an exponentially convergent observer in conjunction with an exponentially stabilizing state feedback control law can guarantee stability of the closed loop system. However, ignoring saturation in the controller output, it can be shown that the closed-loop system is input-to-state stable with the observer error as input, so that stability is preserved under certainty equivalence control (Aamo et al., 2004).

\section{LABORATORY EXPERIMENTS}

Realistic tests of control structures for gas-lift wells are performed using the gas-lift well laboratory setup at TU Delft ${ }^{3}$. The laboratory is shown in Figure 4.

\footnotetext{
3 The experimental setup is designed and implemented by Shell International Exploration and Production B.V., Rijswijk, and is now located in the Kramers Laboratorium voor Fysische Technologie, Faculty of Applied Sciences, Delft University of Technology.
}

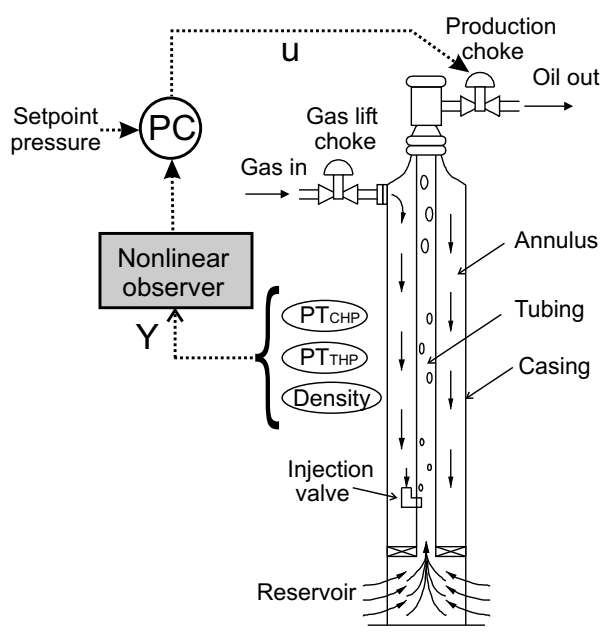

Fig. 3. Control structure applying a nonlinear observer for stabilization of a gas-lift well.

Results from prior experiments show that stabilization of the multiphase flow in the tubing can be achieved by a conventional PI controller adjusting the production choke opening based on measurement of the downhole pressure. The control structure applied in the laboratory experiments controls the estimated downhole pressure, given by the observer, and manipulates the opening of the production choke. The control structure is given in Figure 3.

\subsection{Experimental Setup}

The laboratory installation represents a gas-lift well, using compressed air as lift-gas and water as produced fluid. The production tube is transparent, facilitating visual inspection of the flow phenomena occurring as control is applied. The production tube measures $18 \mathrm{~m}$ in height and with an inner diameter of $20 \mathrm{~mm}$, see Figure $4 \mathrm{a}$. The fluid reservoir is represented by a tube of the same height and an inner diameter of $80 \mathrm{~mm}$. The reservoir pressure is given by the static height of the fluid in the reservoir tube. A 30 litre gas bottle represents the annulus, see Figure 4b, with the gas injection point located at the bottom of the production tube. In the experiments run in this study, gas is fed into the annulus at a constant rate of $11 \mathrm{~L} / \mathrm{min}$ (atmospheric conditions). Input and output signals to and from the installation are handled by a microcomputer system, see Figure $4 \mathrm{c}$, to which a laptop computer is interfaced for running the control algorithm and presenting output.

\subsection{PI Controller and Observer}

The estimated downhole pressure of the gas-lift well was stabilized using the digital PI controller 


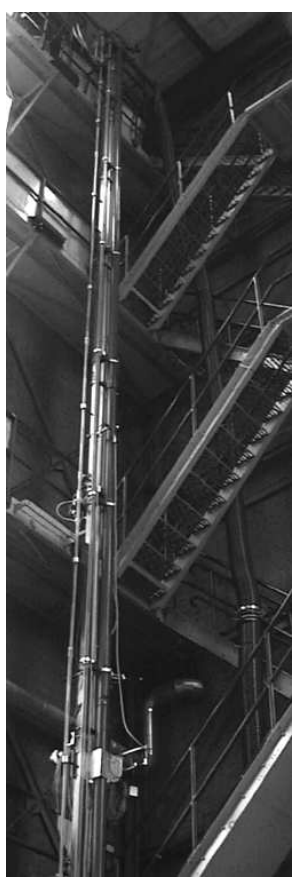

a) The production tube and the reservoir tube.

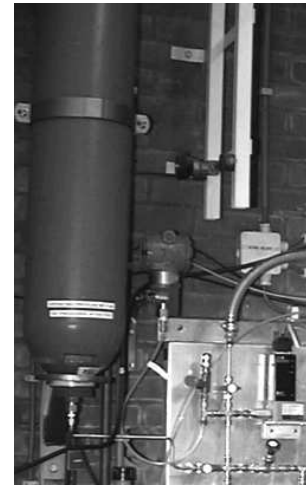

b) The annulus volume

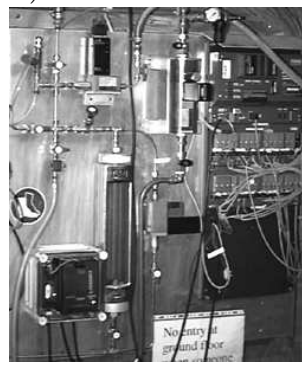

c) The microcomputer
Fig. 4. The gas-lift laboratory

$$
\Delta u_{k}=K_{c}\left[\left(e_{k}-e_{k-1}\right)+\frac{\Delta t}{\tau_{I}} e_{k}\right] .
$$

The gains and integral times applied by the controller are given in Table 1. The observer gains applied in the experiments are given in Table 2.

Table 1. Gain scheduling

\begin{tabular}{|c|c|c|}
\hline Valve Opening & Gain & Integral Time \\
\hline $55 \% \leq u<65 \%$ & -0.5 & $150 \mathrm{sec}$ \\
\hline $65 \% \leq u<73 \%$ & -1.5 & $150 \mathrm{sec}$ \\
\hline $73 \% \leq u<100 \%$ & -2.0 & $200 \mathrm{sec}$ \\
\hline
\end{tabular}

Table 2. Gains - Observer

\begin{tabular}{|c|c|}
\hline Total mass of gas, $c_{1}$ & Total mass, $c_{2}$ \\
\hline 0.5 & 0.01 \\
\hline
\end{tabular}

\subsection{Experimental Results}

The laboratory experiment followed the control sequence given in Table 3 . The multiphase flow is initially open loop stabilized by applying a small choke opening, causing the pressure drop to be friction dominated. When steady-state is reached the controller is turned on, which gradually increases the choke opening and moves the system into the unstable domain. When steadystate again is reached, this time at a large choke opening, the controller is turned off, leaving the choke with the last controlled opening. This is why oscillations appear at the end of the time series, confirming that the new operating point is indeed in the unstable domain.
Table 3. The control sequence

\begin{tabular}{|c|c|c|}
\hline Time Slot & Control & Valve Opening \\
\hline$(-5) \mathrm{min}-0 \mathrm{~min}$ & open loop & $55 \%$ \\
\hline $0 \mathrm{~min}-50 \mathrm{~min}$ & closed loop & controlled \\
\hline $50 \mathrm{~min}-55 \mathrm{~min}$ & open loop & $83.2 \%$ \\
\hline
\end{tabular}

The estimated downhole pressure from the observer and the measured downhole pressure is given in Figure 5. The results show that the downhole pressure given by the observer can be applied to stabilize the laboratory gas-lift well. The controller stabilizes the system at a production choke opening of $79 \%$. After 43 minutes a large external disturbance to the gas supply source is introduced to the system, as can be seen from the peaks in the experimental results. The controller is able to handle this disturbance.
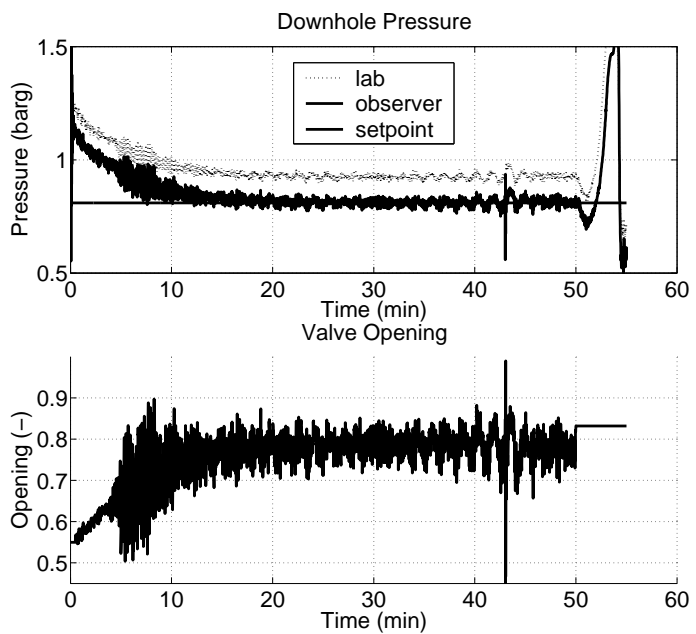

Fig. 5. The downhole pressure given by the observer, the measured downhole pressure and the opening of the production choke.

The measurements applied in the observer, the casing head pressure, the tubing head pressure and the fluid density, are given in Figure 6 and 7. The measurements of the tubing head pressure and the density are low-pass filtered, using a cutoff period of $T_{c, T H P}=20$ seconds and $T_{c, \rho}=10$ seconds, respectively.

The flow rate from the well is given in Figure 6 . The production is achieved with an average production choke opening of $79 \%$. The achieved liquid production at several production choke openings are given in Figure 8. The curve shows the liquid production achieved by open loop production. For the valve openings $55 \%$ to $70 \%$, the production is open loop stable. From $70 \%$ to $100 \%$ valve opening the production is open loop unstable, and is significantly lower compared to the production at $70 \%$ valve opening.

The main objective is to compare the production increase, gained by the use of feedback control, with the maximum stable production achieved without control (70\% valve opening). The increase 

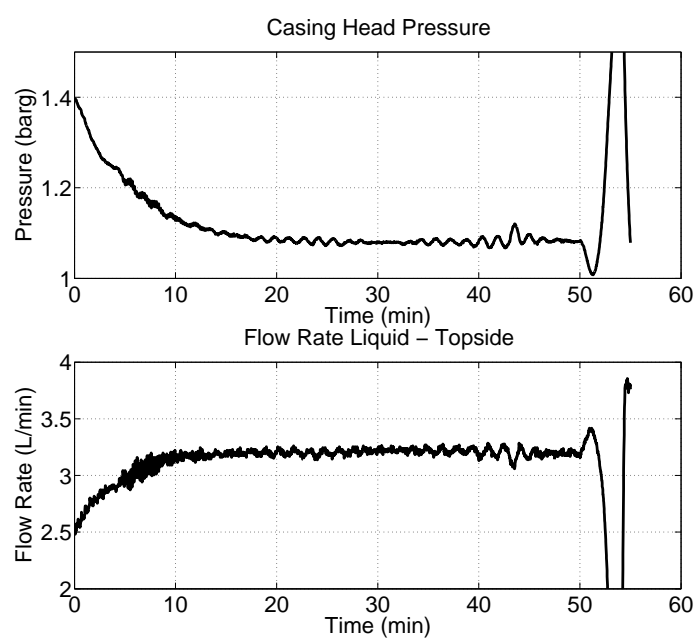

Fig. 6. The pressure in the annulus and the total fluid production.
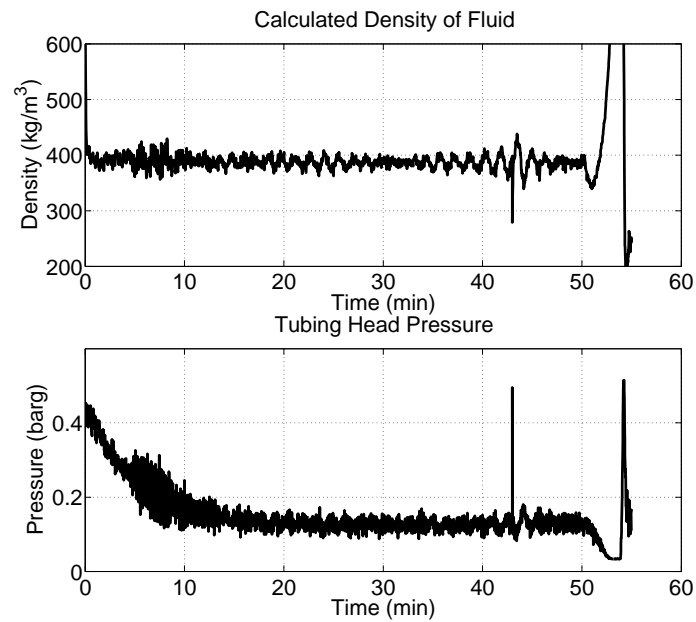

Fig. 7. The fluid density and the pressure in the top of the tubing.

in produced fluid gained by stabilizing the gas-lift well at $79 \%$, by feedback control, is $5 \%$.

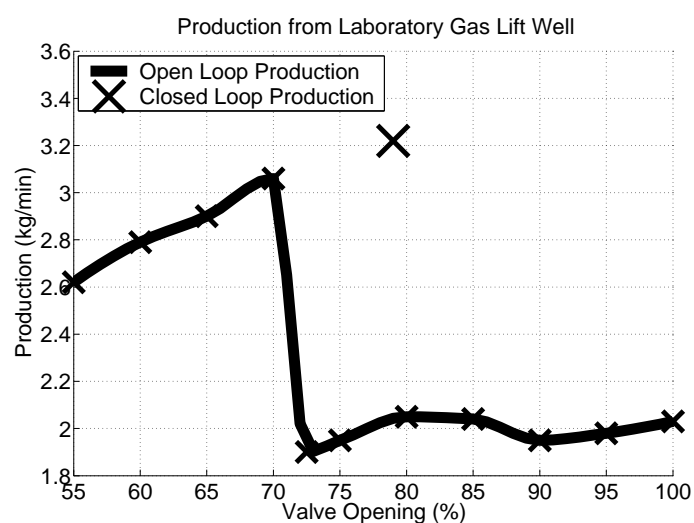

Fig. 8. Production achieved from the gas-lift laboratory by closed loop compared to open loop production.

\section{CONCLUSIONS}

In this paper, we have employed a reduced order nonlinear observer for the states of the multiphase flow in the gas-lift well, and showed in experiments that it can be used in conjunction with a PI controller to stabilize the gas-lift well. The observer, and thereby the entire control scheme, relies on topside measurements only, which is a key feature for the applicability of the scheme. The experimental results presented here, show that an increased production of $5 \%$ is achieved for the gas-lift well laboratory by means of feedback control.

The results of this paper clearly show that there is a potential for increasing production from gas-lift oil wells by installing a relatively simple control system.

\section{ACKNOWLEDGEMENTS}

We gratefully acknowledge the support from Shell International Exploration and Production B.V., and in particular Dr. Richard Fernandes. And Kramers Laboratorium voor Fysische Technologie, Faculty of Applied Sciences, Delft University of Technology, and in particular Prof. Dr. R.V.A. Oliemans.

\section{REFERENCES}

Aamo, O.M., G.O. Eikrem, H. Siahaan and B. Foss (2004). Observer design for gas lifted oil wells. Proceedings of the 2004 American Control Conference.

Dalsmo, M., E. Halvorsen and O. Slupphaug (2002). Active feedback control of unstable wells at the brage field. SPE paper no. 77650 .

Eikrem, G.O., B. Foss, L. Imsland, B. Hu and M. Golan (2002). Stabilization of gas lifted wells. In: Proceedings of the 15th IFAC World Congress. Barcelona, Spain.

Eikrem, G.O., L. Imsland and B. Foss (2004). Stabilization of gas lifted wells based on state estimation. In: Proceedings of the International Symposium on Advanced Control of Chemical Processes. Hong Kong, China.

Imsland, L. (2002). Topics in Nonlinear Control: Output Feedback Stabilization and Control of Positive Systems. PhD thesis. Dept. of Eng. Cybernetics, Norwegian Univ. of Sci. and Tech.

Jansen, B., M. Dalsmo, L. Nøkleberg, K. Havre, V. Kristiansen and P. Lemetayer (1999). Automatic control of unstable gas lifted wells. SPE paper no. 56832.

Kinderen, W.J.G.J. and C.L. Dunham (1998). Real-time artificial lift optimization. SPE $p a-$ per no. 49463.

Xu, Z.G. and M. Golan (1999). Criteria for operation stability of gas lift. SPE paper no. 19362. 